\title{
Exonic Disruption Facilitates Antiviral CRISPR-Cas9 Activity for Multistrain HIV-1 Elimination
}

Jonathan Herskovitz ${ }^{\mathrm{a}, *, 1}$, Mahmudul Hasan ${ }^{\mathrm{b}, 1}$, Milankumar Patel ${ }^{\mathrm{c}}$, Wilson R. Blomberg ${ }^{\mathrm{d}}$, Jacob D. Cohen ${ }^{c}$, Jatin Machhic, Daniel Stein ${ }^{c}$, Evan A. Schroder ${ }^{c}$, JoEllyn McMillan ${ }^{c}$, Channabasavaiah B. Gurumurthyc,e ${ }^{\mathrm{c}}$, Bhavesh D. Kevadiya ${ }^{\mathrm{c}}$, and Howard E. Gendelman ${ }^{\mathrm{a}, \mathrm{b}, \mathrm{c},{ }^{*}}$

${ }^{a}$ Department of Pathology and Microbiology, ${ }^{b}$ Department of Pharmaceutical Sciences, ${ }^{c}$ Department of Pharmacology and Experimental Neuroscience, and ${ }^{\mathrm{e} M o u s e}$ Genome Engineering Core facility, University of Nebraska Medical Center, Omaha, NE 68198

${ }^{d}$ School of Medicine, Creighton University Medical Center, Omaha, NE 68124

${ }^{1}$ Co-first authors

*To whom correspondence should be addressed. Howard E. Gendelman (lead contact for review and correspondence); Jonathan Herskovitz (shared correspondence)

Email: hegendel@unmc.edu; jonathan.herskovitz@unmc.edu

Author Contributions: J.H. and M.H. devised experimental approach; J.H, M.H., M.P., W.B., J.D.C, D.S., and E.A.S. performed experiments. C.B.G., B.D.K., and J.M. provided technical and experimental guidance; J.H. and M.H. prepared figures, legends, and manuscript; and H.E.G provided overall project guidance, research infrastructure, funding, writing and review of the manuscript.

Competing Interest Statement: J.H., M.H., and H.E.G. are named inventors on provisional patents for the CRISPR therapy described in this report $(62 / 985,392 ; 62 / 986,216)$. J.H., M.H., B.K., and H.E.G hold a patent on a virus-like particle-based delivery for HIV-1 CRISPR therapeutics (Docket No. 19040PCT; Serial No. PCT/US2020/016126; International Publication No. WO 2020/160418 A1). H.E.G is a member of the scientific advisory board at Longevity Biotech and a co-founder of Exavir Therapeutics, Inc.

Classification: BIOLOGICAL SCIENCES; Microbiology

Keywords: HIV, CRISPR, tat, lentivirus, elimination 


\begin{abstract}
A barrier to HIV-1 cure rests in the persistence of proviral DNA in infected CD4+ leukocytes. The high mutation rate of HIV-1 gives rise to numerous circulating strains with increased capacity for immune evasion and antiretroviral drug resistance. To facilitate viral elimination while accounting for this diversity, we propose genetic inactivation of proviral DNA with CRISPR-spCas9. We designed a library of "mosaic gRNAs" against a HIV-1 consensus sequence constructed from 4004 clinical strains, targeting the viral transcriptional regulator tat. Testing in 7 HIV-1 transmitted founder strains led, on average, to viral reductions of $82 \%$ with tandem TatD and TatE (TatDE) treatment. No off-target cleavages were recorded. Lentiviral transduction of TatDE attenuated latency reversal by $94 \%$ in HIV-infected, transcriptionally silent ACH2 T cells. In all, TatDE guide RNAs successfully disrupted 5 separate HIV-1 exons (tat 1 -2/rev1-2/gp41) providing a pathway for CRISPR-directed HIV1 cure therapies.
\end{abstract}

\title{
Significance Statement
}

Over 38 million individuals worldwide are infected with HIV-1, which necessitates lifelong dependence on antiretroviral therapy (ART) to prevent viral replication that leads to AIDS. Efforts to rid hosts of HIV-1 are limited by the virus' abilities to integrate proviral DNA in nuclei, mutate their genomes, and lay dormant for decades during ART treatment. We developed mosaic guide RNAs, TatD and TatE, for CRISPR-Cas9 that recognize the majority of known HIV-1 strains and inactivate $94 \%$ of proviral DNA in latently infected cells. Tandem TatDE-CRISPR inactivation of 5 viral exons (tat1-2, rev1-2, and gp41), which blocked HIV-1 replication for 28 days in CD4+ T cells without unwanted editing to the host genome, may serve as a viable strategy for HIV cure.

\section{Main Text}

\section{Introduction}

Defining the scientific pathways required to eliminate the human immunodeficiency virus type one (HIV-1) from its infected human host remains a global health concern for 38 million infected people. To avoid HIV-1 reactivation from latent integrated proviral DNA in CD4+ T cells and mononuclear phagocytes, lifelong antiretroviral therapy (ART) is required. ART protects against virus-induced reductions in numbers and function of CD4+ $T$ lymphocytes and progression to the acquired immunodeficiency syndrome (AIDS) (1). Thus, a functional cure for HIV-1 is needed in order to eliminate dependence on lifelong ART.

While curative measures for HIV-1 are under development, gene editing stands alone in having the ability to remove virus independently from host antiviral adaptive immunity. Although concurrent viral elimination strategies that include broadly neutralizing antibodies (bnAbs) (2), immune modulation (3), and chimeric antigen receptor (CAR)-T cells $(4,5)$ show promise in mice, selection for bnAb resistant strains (6), viral rebound $(3,7)$, and adverse side effects (8) have been reported in monkeys and humans. Treatments notably depend on host FcR-mediated cellular killing or antigen presentation in MHC-I and, as such, rely on host immunity for viral clearance. Thus, gene therapy offers the ability block propagation of HIV-1 infection by directly targeting either host cell receptors or HIV-1 proviral DNA itself. The viral co-receptor CCR5 can be ablated ex vivo (9, 10) while latent viral DNA can be excised in live animals (11). Based on these results, gene therapy for HIV-1 elimination is under active clinical investigation (e.g. NCT02140944, NCT03164135, NCT03666871).

Nonetheless, applying gene therapy towards HIV-1 cure strategies has met several obstacles. One of the most significant rests in viral DNA sequence diversity. With a mutation rate of 1 in 1700 nucleotides (12), HIV-1 quasispecies easily accumulate during the course of viral infection (13). Divergent strains with increased replicative fitness evade host immunity in individuals and circulate through populations (13) as evidenced by the emergence of 11 global HIV-1 subtypes 
(also referred to as clades; A-L) (14). HIV-1 subtypes B and C are the most prevalent clades in Europe and the Americas (10.2\%) (15) and worldwide (46\%) (16), respectively. Thus, gene therapies designed to broadly target then eliminate HIV-1 must overcome strain diversity.

We posit that clustered regularly interspaced short palindromic repeat (CRISPR) approaches can meet the challenge of viral diversity. Potential proviral DNA targets of the 10 kilobase pair (kb) HIV-1 genome include the viral promoter long terminal repeat (LTR) (17-20) and viral transcription factor tat (21-23). CRISPR gene editing can cleave two alternatively spliced HIV1 tat exons. Thus, CRISPR can simultaneously cleave rev and env that are transcribed from the same portion of the genome but in alternate open reading frames (21).

Defining the optimal gRNAs, alone or in combination, to eliminate HIV-1 infection and implementing the approach towards human trials is a challenge. First, computational assessments suggest that proviral targeting choices require affirmation in patient derived HIV-1 isolates. Indeed, previously published LTR-directed gRNAs likely possess limited cleavage efficiencies during natural infection (24). Second, gRNAs must inactivate HIV-1 transcription precluding the assembly of functional virions en trans from intact viral operons. Third, optimal combination gRNAs must minimize any emergence of CRISPR-resistant escape mutants $(20,23)$. While multiplexed CRISPR-Cas9 treatments were designed to account for HIV-1 strain variation (25) and minimize viral escape (21), testing against transmitted founder viral strains was not completed. Fourth, a CRISPR-Cas9 delivery system seeking to find and eliminate virus from its natural target cells must be employed.

Accepting each of these needs, we developed then characterized a CRISPR-Cas 9 system capable of eliminating diverse proviral HIV-1 strains. The approach taken mirrors the "mosaic" HIV1 vaccine design currently in late phase clinical trials (NCT03964415, NCT03060629) serving to immunize those persons with a high risk of acquiring infection with HIV-1 gag/pol/env antigens encoded from global composite of viral strains (26). The data offered supports the concept that CRISPR-Cas9 gRNAs designed against a multiclade HIV-1 consensus sequence, hereafter termed "mosaic gRNAs", can prove effective. The mosaic gRNAs targeting genetically conserved and overlapping tat/rev exons maximized CRISPR-based attenuation of HIV-1 infection. A lentiviral transduced CRISPR-Cas9 system proved successful in its ability to block HIV-1 reactivation from latently infected CD4+ T cells. The data, taken together, brings the approach one step closer towards defining a workable strategy for HIV-1 cure.

\section{Results}

In silico design of HIV-1 tat mosaic gRNAs. Targeted HIV-1 gene therapy requires specific recognition of diverse proviral sequences. HIV-1 sequence diversity occurs both at the population level and during disease progression, thus lessening the effectiveness of host immune responses and promoting the emergence of antiretroviral drug resistance (27). While the 5' LTR is a promoter for viral transcription and is susceptible to CRISPR-based HIV-1 inactivation, we reasoned that targeting the driver of LTR-directed full-length transcription, HIV-1 tat, would improve CRISPR therapeutic efficacy. To test this notion, a multiple sequence alignment heat map, constructed from 4004 HIV-1 strains, was utilized to observe the degree of nucleotide heterogeneity across the viral genome (Figure 1). Conserved regions of the HIV-1 genome include portions of flanking LTRs, gp120-encoding segments of env, and multiple exon overlaps (Figure 1A). Low entropy in HIV-1 genes encoding p6/protease, tat/rev, vpu/gp120, and nef/3' LTR highlight sequence preservation in loci that govern multiple phases of the viral lifecycle.

As comparators, two CRISPR-Cas9 reference systems were adopted. First, LTR-1 and GagD are gRNAs that target the U3 portion of LTR and MA region of gag, respectively (Figures 1B, S1). LTR-1/GagD was selected as a reference control because of its utility in sterilizing onethird of HIV-1 infected humanized mice, thereby establishing proof-of-concept for a CRISPR-based HIV cure in an animal model (11). Second, DLTR-1 and DLTR-2 are top-rated gRNAs from a quadruplex panel designed to cleave most known HIV-1 strains with minimal off-target human genome recognition (25). Delivery of DLTR-1 and DLTR-2 gRNAs to cells remains a challenge, 
however, because their targeting of HIV-1 LTR (Figure S1) simultaneously also thwarts the synthesis of all CRISPR-transducing lentiviruses. Control gRNAs display complete 20 base pair (bp) recognition of $11-30 \%$ of curated HIV-1 strains (Table S1). A minimum off-target threshold of 2.266 was set for tat gRNA design in accordance with a CRISPR-specificity algorithm score for the DLTR-2 control.

A library of eight HIV-1 tat-targeting gRNAs designed for deployment with Streptococcus pyogenes Cas9 (spCas9) endonuclease were created. These "mosaic" gRNAs were constructed against the tat consensus sequence synthesized from $4004 \mathrm{HIV}-1$ strains (Figure S1). Five of the eight produced gRNAs target the sense strand (Figure 1C). Because portions of tat overlap with rev and the gp41 portion of env, up to three exons could be simultaneously disrupted with these gRNAs (Figure S2). Notably, tat-directed gRNAs retain full 20 bp complementarity for $6-67 \%$ of all known HIV-1 strains (Table 1). Duplexed mosaic gRNA treatments were found to target at least $56-62 \%$ of strains, assuming no nucleotide mismatch tolerance (Table S2). Taken together, these data demonstrate that gRNAs can be produced with broad HIV-1 excision potential while, at the same time, limiting off-target effects.

A

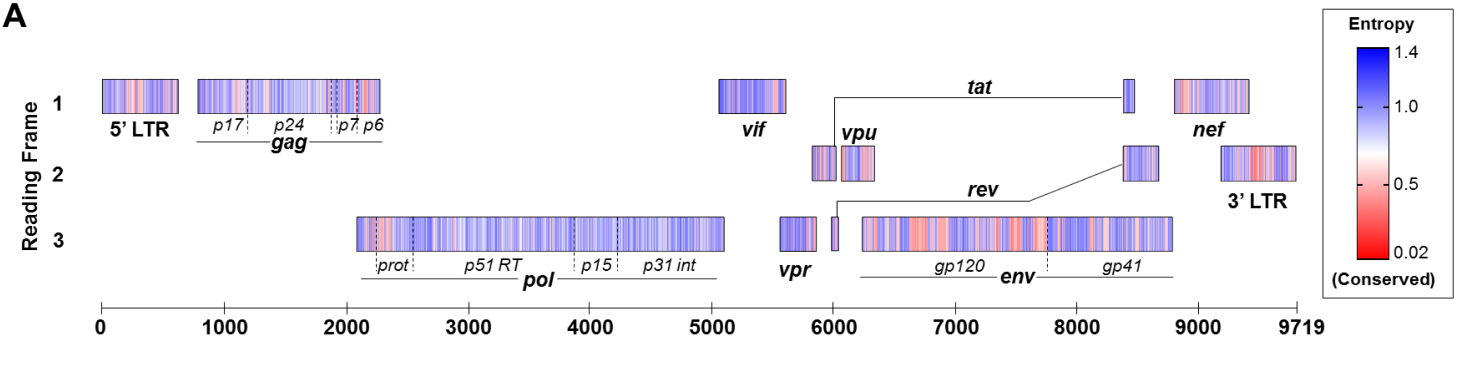

в

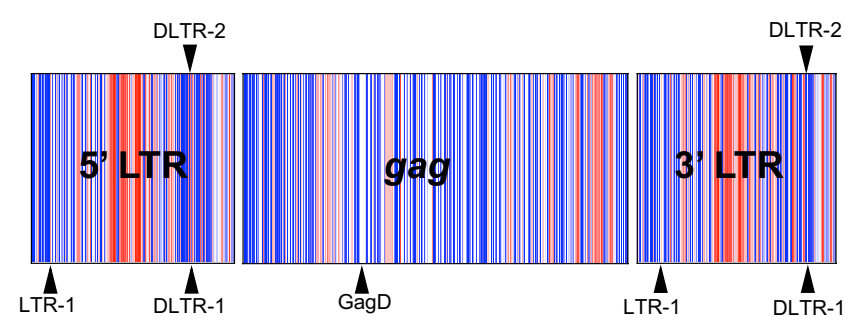

C
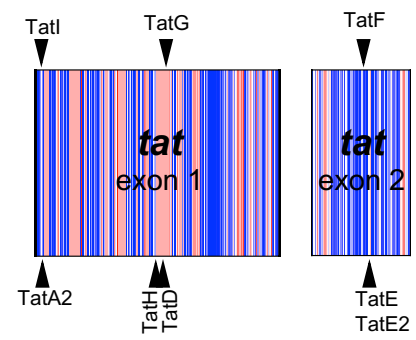

Figure 1. HIV CRISPR-Cas9 Mosaic gRNA Design. (A) Nucleotide heterogeneity of 4004 annotated HIV-1 strains is depicted in heat-map form demonstrating entropic (blue) or conserved (red) loci in all 3 reading frames. Previously reported gRNAs against LTR- and gag regions, used herein as reference controls $(B)$, or novel gRNAs targeting mosaic tat sequences of HIV-1 $(C)$ were designed. gRNAs against antisense or sense sequences are shown as downward or upward facing arrows, respectively. 
Tat/rev-directed gRNAs suppress HIV-1 replication. To test the hypothesis that conserved gRNAs would most effectively limit viral replication, a plasmid co-transfection screen was implemented. Human embryonic kidney (HEK293FT) cells were transfected with plasmids encoding HIV-1 in the absence (untreated control) or presence of spCas9-gRNA constructs. Seven HIV-1 molecular clones, one laboratory (NL4-3) plus six clade B founder strains, were included to reflect predominant North American and European viral subtype sequence heterogeneity. Four independent co-transfection screens were performed to ensure experimental accuracy. Supernatants were measured after 72 hours for HIV reverse transcriptase (RT) activity.

The single mosaic gRNA CRISPR TatE and TatD constructs demonstrated the greatest suppression of HIV-1 replication (Figure 2A). TatE gRNA reduced RT activity by an average of $76 \%( \pm 6.5 \%$, standard error of the mean (SEM)), with more than $75 \%$ reduction in 6 of 7 tested HIV-1 strains. Notably, TatE outperformed all single gRNA controls, including DLTR-2. Pearson correlation analysis evaluated whether CRISPR gRNA activity was associated with target sequence conservation (Figure 2B). While positive trends were observed, significance was achieved when TatE was excluded from analysis. The data support the idea that although gRNA targeting conserved genomic regions of HIV-1 proviral DNA are generally more efficacious, the TatE locus is critical to viral replication.

We next sought to determine whether proviral DNA excision induced by two CRISPR-Cas9 gRNAs would further suppress HIV-1 replication. The noted efficacy of TatE, which targets a region of tat that overlaps with both rev and env, prompted us to posit that simultaneous disruptions of multiple viral exons would most drastically blunt HIV-1's lifecycle. Plasmids were subcloned to express various combinations of the top three performing tat-directed gRNAs. Table S2 summarizes the percent conservation, excision fragment length, and number of deactivated exons elicited by each pairing. Increased antiretroviral activity was observed when TatD and TatE gRNAs were co-expressed (TatDE). Viral replication in TatDE-treated cells were lowered on average by $82 \%( \pm 4.6 \%$ SEM), with a range of $65-98 \%$ across all tested viral strains (Figure $2 \mathrm{~A})$. TatDE demonstrated greater levels of viral suppression compared to LTR-1/GagD ("LG") and DLTR1/DLTR-2 ("DLTR1-2") duplexed reference CRISPR controls by measured RT activity. The $\sim 2.5$ kilobase pair $(\mathrm{kb})$ dropout of intervening proviral DNA was observed against all seven HIV-1 molecular clones (Figures 2C, S3). To confirm the accuracy of TatDE CRISPR, excision bands were Sanger sequenced and analyzed by Inference of CRISPR Edits (ICE) algorithm (Figure S4). Sequencing data qualitatively depict nucleotide degeneracy in both target loci, with insertion/deletion (indel) mutations at a rate of $25-51 \%$ in excision bands. Pairing our two most conserved efficacious gRNAs, TatDH, and of our largest excising duplex, TatEH, proved inferior to 5-exon inactivating TatDE in suppressing RT activity. Taken together, these data support the hypothesis that disrupting a maximal number of viral exons by CRISPR-Cas9 leads to the greatest suppression of HIV-1 replication. 
A

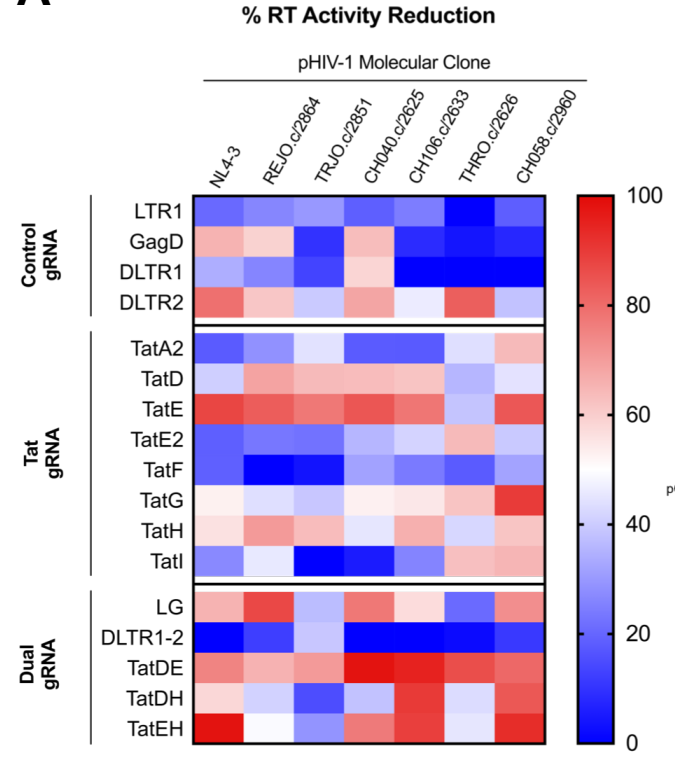

D

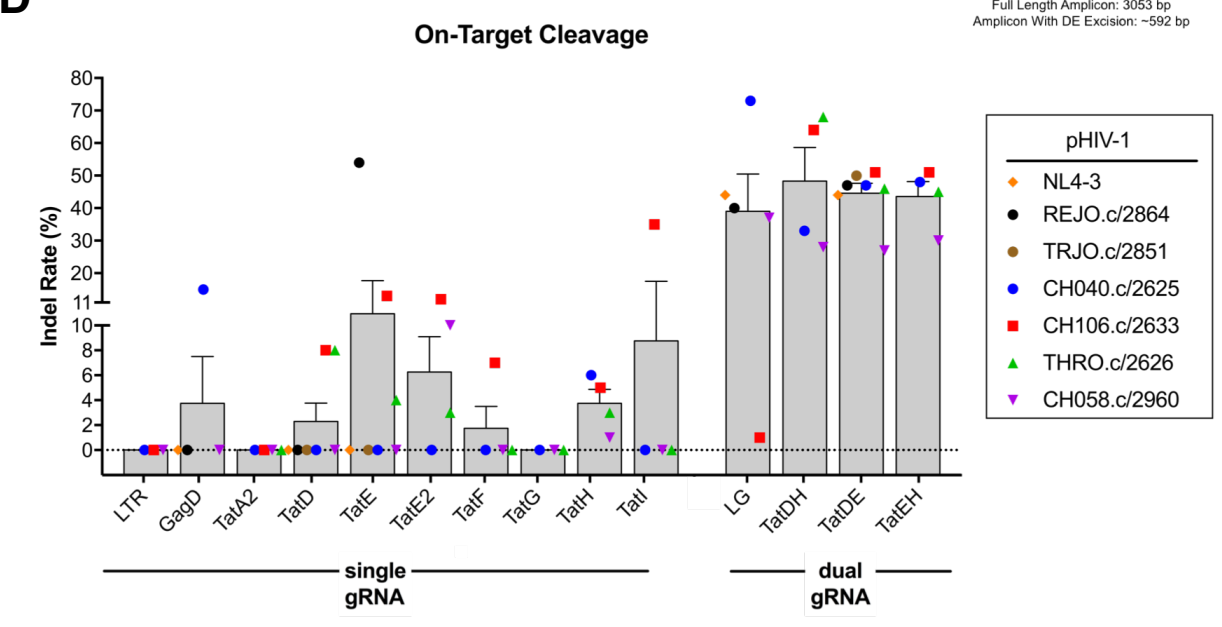

B
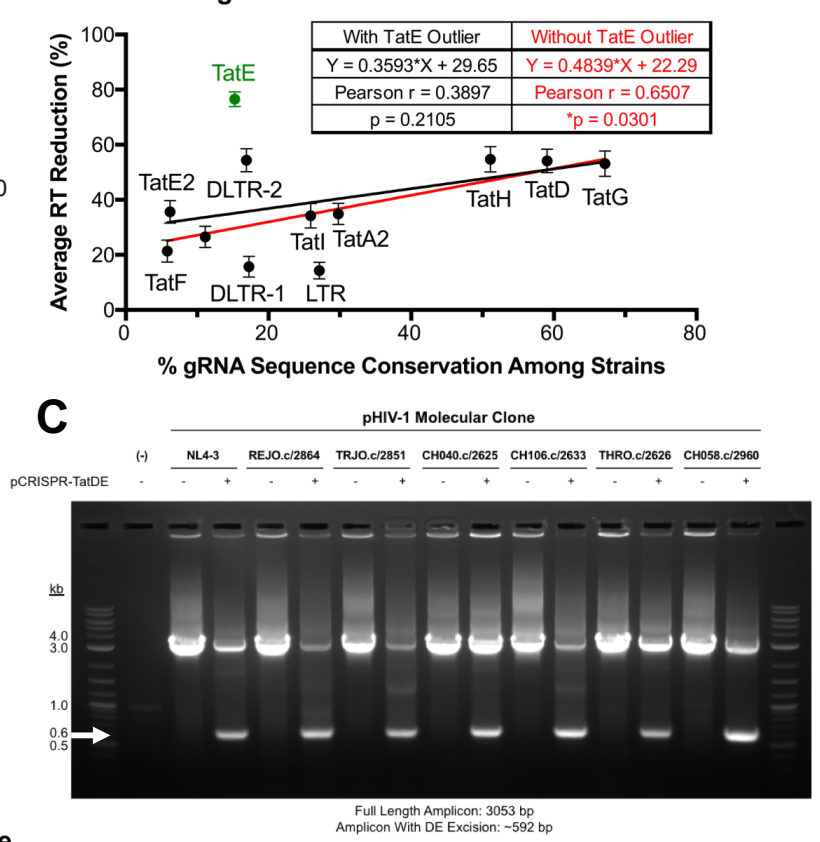

Figure 2. TatDE Dual gRNAs Facilitate Multistrain HIV-1 Excision. (A) A gRNA library was screened against multiple HIV-1 molecular clones via co-transfection of HEK293FT cells for reduction in viral replication as measured by reverse-transcriptase (RT) activity assay. $(B)$ Pearson correlation between gRNA target conservation among 4004 proviral DNA sequences and average RT activity knockdown was assessed. (C) PCR was performed on DNA extracted from PCR amplified untreated- or CRISPR-TatDE plasmid-treated cells. White arrow indicates expected molecular size of TatDE excision bands. (D) PCR reaction contents were Sanger sequenced and subjected to Inference of CRISPR Edits v2.0 (ICE, Synthego 2020) to visualize nucleotide editing in the PAM/protospacer region. Data in $(A-B, D)$ depict mean \pm SEM from four independent experiments each containing biological triplicates. 
On-target CRISPR-Cas9 TatDE specificity. We next evaluated the specificity of our tat-directed CRISPR-Cas9 by comparing the degree of proviral genome editing to that observed in off-target loci. ICE analysis performed on Sanger sequences from these reactions revealed that dual gRNA systems improve indel mutation rates to $>40 \%$ as compared to $<10 \%$ seen with single gRNA controls (Figure 2D). TatDE therapy resulted in an average of $45 \%( \pm 3.0 \%$ SEM) gene modification rate with $40-50 \%$ editing in 6 of 7 tested strains, supporting TatDE's ability to deactivate a broad variety of HIV-1 proviral species.

To ensure TatDE cleavage is restricted to HIV-1 proviral DNA, we investigated the potential for aberrant indels at off-target loci in the human genome. These covered 5 possible loci recognized by each TatD, TatE, LTR-1, and GagD. At all putative off-target positions for TatD and TatE, no editing was observed (Table S3). This was sustained regardless of whether cells were treated with single or dual gRNAs. Similarly, off-target analysis by ICE for LTR-1 and GagD failed to demonstrate indel mutations in host genes (Table S4). Seven of 10 possible off-target regions for TatDE fell in intronic regions whereas only 2 of 10 for LTR-1/GagD were noncoding. These results reinforce the notion that TatDE CRISPR is not more likely to affect host gene expression than other HIV-1 CRISPR systems.

TatDE CRISPR-Cas9 eliminates latent proviral HIV-1. Having demonstrated the ability of TatDE CRISPR to suppress plasmid-encoded viral transcription, we next investigated whether latent HIV1 could be excised in leukocytes. For these experiments, TatD, TatE, or non-targeting control gRNAs were cloned onto a lentiviral-CRISPR expression plasmid. To first assess CRISPR-Cas9 function, $\mathrm{ACH} 2 \mathrm{~T}$ cells and $\mathrm{U} 1$ promonocytes bearing 1-2 copies of latent HIV-1 proviral DNA copies per cell were transfected with cloned lentiviral-CRISPR constructs. Subsequent stimulation of ACH2 T cells with TNFa induced viral rebound in control- and LTR-1/GagD-treated cultures but not in those receiving single- or dual TatD plus TatE treatments (Figure S5). U1 promonocytes were equally responsive to LTR-1/GagD and TatD/TatE treatments (Figure S6). HIV-1 proviral DNA excision was present in both cell types and confirmed by sequencing. These data in aggregate validate the TatDE CRISPR-Cas9 system for lentiviral delivery.

We next ascertained whether lentiviral transduction of TatDE CRISPR-Cas9 could halt HIV-1 induction from latently infected $A C H 2 T$ cells. Transgene expression was measured by reverse-transcriptase quantitative PCR (RT-qPCR) and determined to be above detection limits (Figure 3A). Dual TatD/TatE lentiviral treatments blocked TNFa-induced stimulation. Cotransduction of TatD/TatE at MOls of 10 and 1 significantly reduced the release of HIV-1 into culture supernatants by $80 \%$ and $94 \%$, respectively (Figures 3B-C). We also evaluated cell DNA by nested PCR for TatDE HIV-1 excision (Figures 3E, S7). A predictable $\sim 525$ bp excision band was present for cells co-transduced at a MOI of 1 , supporting the reduction in RT activity at this MOI. Shorter amplicons were observed with $\mathrm{MOI} 0.1$ treatment, however the shortened amplicons disappeared in stimulated conditions. Paired with the RT activity results (Figure 3D), it is likely that transduction at a low MOI was subtherapeutic, as few cells bear the CRISPR-induced indel. Conversely, we cannot exclude the possibility that the efficacy observed at MOI 10 in the absence of excision bands results from cytopathicity encountered with high levels of lentiviral treatment. These results cumulatively support the utility of lentiviral delivery of TatDE CRISPR-Cas9 in eliminating latent proviral HIV-1. 
A

B

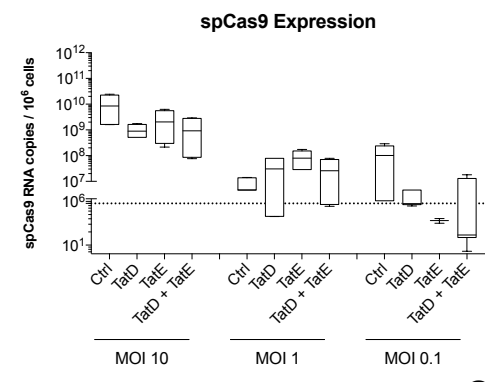

MOI 10



— Lenti-CRISPR-gRNA-
E

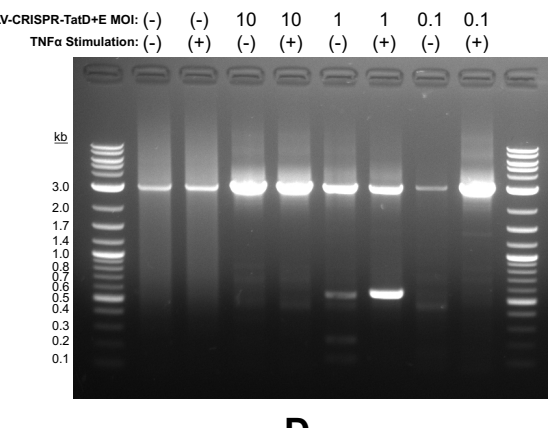

MOI 1



— Lenti-CRISPR-gRNA -
D

MOI 0.1

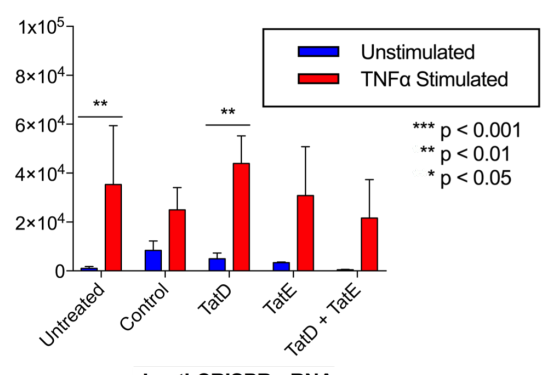

— Lenti-CRISPR-gRNA-

Figure 3. Lentiviral TatD + TatE CRISPR co-transduction Inactivates Latent HIV-1. ACH2 T cells bearing 1 copy of HIV-1 proviral DNA were transduced with lentivirus bearing spCas9-gRNA transgene at multiplicities of infection (MOI) 10, 1, or 0.1 . After 72 hours, cells were stimulated with TNFa $(15 \mathrm{ng} / \mathrm{mL}$ ) for 72 additional hours. $(A)$ spCas 9 expression as measured by RT-qPCR. $(B-D)$ RT activity assay from culture supernatants. $(E)$ Nested PCR for proviral DNA excision wherein unedited amplicons are $2986 \mathrm{bp}$ and CRISPR-edited amplicons are approximately $525 \mathrm{bp}$, depending on insertion / deletion mutagenesis. White arrow indicates expected molecular size in presence of TatDE excision. Significance assessed by two-way ANOVA. 
Exonic disruption of the tatE locus attenuates HIV-1 replication. As a potential mechanism to explain TatE's high antiretroviral activity despite low sequence conservation, we investigated how disruption of different numbers of viral exons affects HIV-1 replicative fitness. Three HIV-1 NL4-3-AnefeGFP non-frameshift point mutants were created to parallel CRISPR mutation profiles (Figure 4A), in which two- $(\Delta T a t D)$, three- $(\Delta T a t E)$, or five $(\Delta T a t D E)$ exons were altered (Figure $4 B$ ). Resulting virions were imaged by transmission electron microscopy (TEM; Figure 4C) and titered by RTqPCR. The size of the HIV-1 tat mutants ranged from 110.6-150.5 nm in diameter, closely approximating that of wildtype HIV-1 measured at $125.4 \mathrm{~nm}$. Likewise, the HIV-1 tat mutants' nearly spherical appearance and inner conical capsid matched the morphology of wildtype control virus. Next, CD4+ T lymphocytes were infected at a MOI of 0.1 . At 10 days following HIV-1 infection, significant differences in RT activity were detected in culture supernatants (Figure 4D). HIV-1NL4-3$\triangle t a t D$ displayed nearly equivalent levels of viral replication as wildtype virus, while mutants bearing $\geq 3$ disrupted exons were significantly lower in RT activity compared to unmutated control. As CD4+ $\mathrm{T}$ cell proliferation rates differed after 10 days due to HIV-1 induced cytopathicity, the percent of cultured HIV-1 infected cells was measured by flow cytometry during a 28-day time course (Figure


HIV-1 NL4-3-StatDE remained at or around baseline for four weeks. These data suggest that the locus targeted by TatE gRNA is critical for maximal CRISPR activity. In aggregate, we conclude that the high efficacy of TatDE CRISPR-Cas9 therapy against numerous HIV-1 strains results from disrupting five viral exons simultaneously.

A

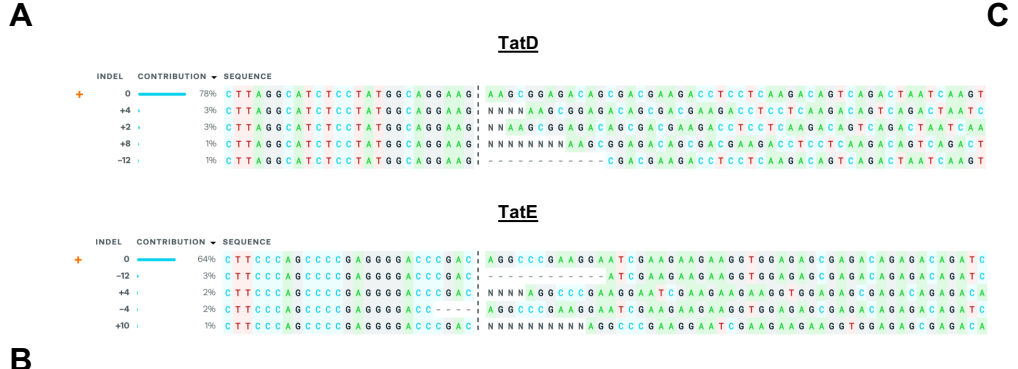

B



c

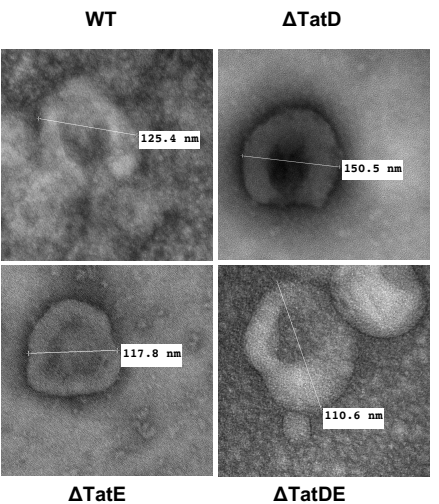

D



E

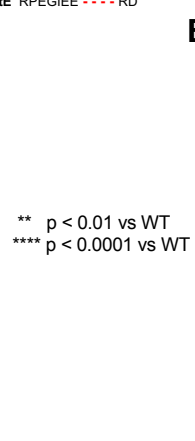

E



Figure 4. Exonic Disruption Compromises HIV-1 Replicative Fitness. ( $A-B)$ Insertion / deletion profiles among the most-efficacious single gRNAs from a co-transfection screen were assessed by Synthego ICE v2.0 algorithm. Highest frequency insertions or deletions were selected for subsequent non-frameshift site-directed mutagenesis of HIV-1 NL4-3-Anef-eGFP encoding plasmid. (C) Transmission electron micrographs of single- or dual-tat mutants. Spherical diameter measurements were taken at the time of imaging (inset). (D-E) CEMss CD4+ T cell lines were 



activity $(D)$ or by flow cytometry for \% GFP-positive cells $(E)$.

\section{Discussion}

Over 79,000 unique human- or simian immunodeficiency virus (HIVISIV) sequences spanning eleven global subtypes have been identified (14). The presence of a $6 \%$ mutation rate even among elite controllers highlights the presence of a multitude of intrapatient HIV-1 quasispecies (28). These considerations led us to design mosaic CRISPR-Cas9 guide gRNAs against conserved and multi-exon regions of a consensus HIV-1 sequence to facilitate viral elimination. Herein, we introduce exonic disruption as a guiding principle for CRISPR therapeutics as a required link to control residual viral infection

Our data establish an association between tat/rev target DNA conservation and CRISPRCas9 efficacy across multiple HIV-1 transmitted founder strains (Figure 2B). The impact of this finding is twofold. First, it validates our mosaic gRNA approach as a tenable means of using CRISPR to inactivate genetically heterogenous viral targets. Second, the correlation reveals that conserved tat/rev-directed gRNAs outperform LTR-based reference controls. Given that these were selected for their potential to recognize divergent HIV-1 quasispecies in patients (25) and facilitate elimination from infected hosts (29), the comparative superiority of TatDE represents an advance in CRISPR therapy as a strategy for HIV-1 elimination.

A second advantage of the TatDE mosaic gRNA approach is that it identifies exonic disruption as a potential mechanism for antiretroviral efficacy. Assessed variables include CRISPR on-target cleavage efficiency, target DNA conservation, excision length, and numbers of disrupted exons during CRISPR-Cas9 treatment. Non-frameshift site-directed mutagenesis of 3 or more exons at the TatE locus, but not the 2 exons at TatD, significantly suppressed viral replication. In our gRNA co-transfection screen, the most broadly active CRISPR treatments included tat/rev/gp41-directed TatE. One implication that follows is that transmission of TatE-directed CRISPR may help overcome low potency $(30)$ and viral escape $(31,32)$ otherwise associated with single gRNA treatment of HIV-1. These results also suggest that cleavage at other multiexon locations along the HIV-1 genome, such as gag/pol and nef/LTR, or tat/rev/gp41 by other Cas nucleases $(20,33)$ may afford broader antiviral coverage. Thus, exonic disruption paired with target DNA conservation appear integral in generating effective HIV-1 CRISPR therapeutics.

Delivery of HIV-1 specific CRISPR payloads remains one final parameter yet to be optimized prior to human administration. Current ex vivo approaches ablate the HIV-1 co-receptor CCR5 with CRISPR-transducing lentiviruses $(34,35)$. However, the $5 \%$ editing efficiency in transduced human hematopoietic stem cells failed to render viral suppression upon antiretroviral drug withdrawal (34). These findings informed our strategy to deploy TatDE CRISPR via a highly efficient viral vector in leukocytes. With lentiviral transduction at a MOI of 1 , the TatDE CRISPR transgene was detected at an average of 33.2 spCas9 RNA copies per AHC2 T cell, with a 94\% reduction in RT activity and clear viral excision (Figure 3). In contrast, a single broad spectrum gRNA targeting LTR transduced at this same level still allowed viral rebound in $\sim 20 \%$ latently infected JLat cells (36). Other studies have achieved $>90 \%$ reduction in HIV-1 reactivation and prevention of viral escape when CRISPR-Cas9 was lentivirally transduced in T cells at MOls 10$15(21,23,37)$. These differences highlight the likelihood that TatDE CRISPR-Cas9 maintains greater potency against HIV-1 compared to other CRISPR-Cas9 therapies.

A possible critique of our vector choice is that patients will likely already be receiving combination antiretroviral drugs that inhibit lentivirus' ability to transduce CRISPR. Nonetheless, latently infected JLat cells, uninfected- and HIV-1-positive patient blood mononuclear cells have been treated successfully by lentivirus transduction in the presence of antiretroviral drugs $(29,37)$. This sets a stage for future optimization of dosage and ART timing to enable CRISPR delivery by lentivirus in HIV-1 infected patients. Phase I clinical trials evaluating the safety of lentiviraltransduced CRISPR-Cas9 are ongoing for B-cell acute lymphoblastic leukemia (B-ALL; 
NCT04557436) and HIV-1 patients in the context of hematological malignancies (NCT03164135). Thus, our ability to potently deactivate HIV-1 with lentiviral vectors may prove essential in avoiding adverse events with TatDE CRISPR therapy.

Selectivity of the CRISPR-Cas system for HIV-1 proviral DNA is a key factor in assessing our treatment's therapeutic window. No off-target edits were observed with TatDE treatment in the 10 loci surveyed by a Sanger sequencing-based algorithm. By contrast, ICE data demonstrate a mean $45 \%$ editing efficiency of proviral DNA template with TatDE CRISPR. This led to detectable excision of $\sim 2.5 \mathrm{~kb}$ of viral DNA and to nearly complete abolition of viral release. These findings are aligned with reports that $\sim 10 \%$ and $39-63 \%$ editing efficiencies from multiplex endonuclease treatments resulted in more than $90 \%$ knockdown in herpes simplex virus and HIV-1, respectively $(23,38)$. Dysregulation of positive feedback loops, as in the case of tat/rev-directed CRISPR-Cas9, and the formation of dominant negative proteins encoded after gene editing may explain the disproportionately high efficacy in each of these cases. The HIV-1 proviral genomes isolated from elite controllers display large deletions of env sequences flanked by tat/rev amongst other regulatory genes (39). It is postulated that defective virus in elite controllers primes the host immune system to clear cells bearing replication-competent HIV-1 proviral DNA (39). Therefore, we posit that TatDE CRISPR therapy may similarly compromise latent HIV-1 sequences thereby inducing immune-mediated clearance in vivo.

Planned studies will serve to address future needs in translating this strategy for human use. TatDE's multistrain potential will be further validated against additional HIV-1 strains and clades for antiviral activity. We will also interrogate the lack of off-target editing within the human genome by employing CIRCLE-seq $(40,41)$, Guide-seq $(36,42)$ and whole genome sequencing (29) to our TatDE CRISPR-treated samples. Finally, we plan to further characterize TatDE's mechanism of action in terms of its ability to downregulate full-length viral transcripts due to tat, diminish rev-associated nuclear export, and impede gp41-mediated viral entry. These additional studies should clarify the value in expanding our conserved, multi-exon targeting CRISPR library to other Cas endonucleases.

To conclude, the combination of "diverse" strain-inactivating TatD and "tri-exon"-directed TatE gRNAs prompted maximal suppression of HIV-1 replication by CRISPR-Cas9. Further refinement of these methodologies may advance the utility of gene therapy in eliminating latent viral infection from infected hosts across the globe.

\section{Materials and Methods}

Detailed protocols for materials and methods are provided in Supplementary Information.

Cloning and transfection. CRISPR plasmid constructs containing mosaic gRNAs were cloned by T4 ligase oligonucleotide insertion (Table S5) in px333 (Addgene \#64073) or pLentiCRISPRRFP657 (Addgene \#75162) and transformed into STBL3 E. coli. In Fusion HD Cloning (Takara \#638920) was utilized to synthesize tat mutants on pHIV-1 NL4-3- $\triangle$ nef-eGFP background. Maxiprep purified plasmids were transfected into HEK293FT cells using PEI or into ACH2 / U1 leukocytes via TransIT-Jurkat reagent (Mirus \#2120). Supernatants were collected 72 hours after media replacement (PEI) or plasmid transfection (TransIT-Jurkat).

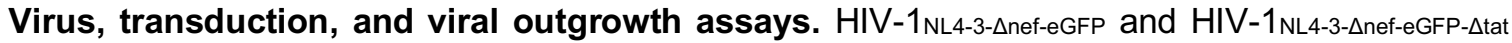
were synthesized by reverse-genetics from supernatants of transfected HEK293FT cells. Supernatants were passed through $0.45 \mu \mathrm{m}$ filters and ultracentrifuged to purify and concentrate virus. HIV-1 viral stocks were titered by RT-qPCR (Takara \#631235) to determine viral RNA copies / $\mathrm{mL}$. Multiplicities of infection (MOI) for HIV-1 infection of CEM-SS T cells (NIH ARP \#776) were calculated as viral RNA copies / cell. CRISPR-transducing lentivirus was commercially prepared at the University of lowa Viral Vector Core Facility and titered using digital droplet PCR. ACH2 T cells (NIH ARP \#349) were transduced with CRISPR-encoding lentivirus at designated MOIs such that MOI 1 = 1 transducing unit per cell. ACH2 and U1 (NIH ARP \#165) were stimulated 3 days post 
transfection or transduction with recombinant tumor necrosis factor alpha (TNFa; $15 \mathrm{ng} / \mathrm{mL}$ ) or phorbol 12-myristate 13-acetate (PMA; $20 \mathrm{ng} / \mathrm{mL}$ ) for 3 additional days followed by experimental harvest.

(RT-q)PCR. DNA was harvested from experimental pooled biological triplicates using NucleoSpin Tissue XS Micro kit (Machery-Nagel \#740901). PCR for proviral DNA excision was run in $25 \mu \mathrm{L}$ reactions using PrimeTime Gene Expression Master Mix (IDT \#1055772) using 150 ng template DNA and 35 cycles or 15 cycles plus 35 cycles for nested PCR. Off-target PCRs were performed with $150 \mathrm{ng}$ template DNA in $25 \mu \mathrm{L}$ reactions using AmpliTaq Gold 360 master mix. Primer sequences for all reactions are provided in Table S6. Gel extracted PCR amplicons and total PCR contents were sequenced and subjected to Synthego Inference of CRISPR Edits (ICE) Analysis v2.0 (https://ice.synthego.com/\#/).

RNA was harvested from pooled biological triplicates using TRIzol ${ }^{\mathrm{TM}}$ solution (Invitrogen \#15596026), DNase I digested (Zymo Research \#E1010), and converted to cDNA (Thermo Scientific \#AB1453B). RT-qPCR was performed in technical triplicates using standard curve absolute quantitation for gapdh ( 1 diploid genome $=6.6$ picograms DNA extracted from ACH2) and spCas9 (pLenti-CRISPR-RFP657; $10^{9}$ copies equal $12.01 \mathrm{ng}$ according to MW $7.22 \times 10^{6} \mathrm{Da}$ ). Reactions were performed in $10 \mu \mathrm{L}$ volumes containing $1 \mu \mathrm{L}$ cDNA templated, $5 \mu \mathrm{L}$ PrimeTime Gene Expression Master Mix, 500 nM of each primer (5'-CCCAAGAGGAACAGCGATAAG-3'; 5'CCACCACCAGCACAGAATAG-3'), and $0.5 \mu \mathrm{L}$ 20x GAPDH endogenous control probe (Applied Biosystems \#4333764T) or $200 \mathrm{nM}$ spCas9 probe (56-FAM/ATCGCCAGA/ZENIAAGAAGGACTGGGAC/3IABkFQ).

Reverse transcriptase activity assay. Culture supernatants were assayed for RT activity by surveying for ${ }^{3} \mathrm{H}$-thymidine incorporation (3H-TTP; Perkin Elmer \#NET221A001MC). Briefly, $10 \mu \mathrm{L}$ of culture supernatants were spiked into 96-well round bottom plates, digested for 15 minutes with $10 \mu \mathrm{L}$ solution A (100 mM Tris- $\mathrm{HCl} \mathrm{pH} 7.9,300 \mathrm{mM} \mathrm{KCl}, 10 \mathrm{mM}$ DTT, $0.1 \% \mathrm{NP}-40)$, then reacted with $25 \mu \mathrm{L}$ solution B (50 mM Tris-HCl pH 7.9, $150 \mathrm{mM} \mathrm{KCl,} 5 \mathrm{mM}$ DTT, $15 \mathrm{mM} \mathrm{MgCl} 2,0.05 \%$ NP40, $0.250 \mathrm{U} / \mathrm{mL}$ oligo dt pd(T)12-18), $10 \mu \mathrm{Ci} / \mathrm{mL} 3 \mathrm{H}-\mathrm{TTP}(4 \mu \mathrm{L} / \mathrm{mL}$; 3H-deoxythimidine 5'triphosphate, tetrasodium salt, (methyl-3H)). After 20 hours incubations, $50 \mu \mathrm{L}$ ice cold $10 \%$ tricholoroacetic acid was added to plates, vacuum filtered across 96-well MicroHarvest Plates, and red by scintillation counting using TopCount Scintillation counter (Perkin Elmer).

Statistical Analyses. Experiments were un with biological replicates $(n=3$ or 4$)$ in at least two separate trials. Data represent mean \pm standard error of the mean (SEM). Pearson correlation, one-way ANOVA with Dunnet correction, and two-way ANOVA with Sidak correction for multiple comparisons were calculated using GraphPad Prism v7.0a for Mac OS X (GraphPad Software, San Diego, California USA, www.graphpad.com).

\section{Acknowledgments}

This work was supported in part by the National Institutes of Health Grants P01 DA028555, P30 MH062261, R01 MH115860, R01 NS034249, R01 NS036126, R01 MH121402, T32 NS105594 (to H.E.G), UNMC Assistantship / Fellowship Program (to J.H. and M.H.), Nicholas Badami Fellowship (to J.H), and the Carol Swartz Emerging Neuroscience Fund (to H.E.G). The authors thank Drs. Beat Bornhauser (U. of Zurich), Kamel Khalili (Temple University), and Won-Bin Young (U. of Pittsburgh) for kindly providing plasmids used in this project. Gratitude is also extended to Nicholas Conoan and the UNMC Electron Microscopy Core for their assistance. A final word of appreciation is directed to Daniel Chadash and the engineering team at Genome Compiler for making open access cloning software freely available online. 


\section{References}

1. Gupta-Wright A, et al. (2020) Virological failure, HIV-1 drug resistance, and early mortality in adults admitted to hospital in Malawi: an observational cohort study. Lancet HIV 7(9):e620-e628.

2. Wu X, et al. (2018) Tandem bispecific neutralizing antibody eliminates HIV-1 infection in humanized mice. J Clin Invest 128(6):2239-2251.

3. Borducchi EN, et al. (2018) Antibody and TLR7 agonist delay viral rebound in SHIVinfected monkeys. Nature 563(7731):360-364.

4. Anthony-Gonda K, et al. (2019) Multispecific anti-HIV duoCAR-T cells display broad in vitro antiviral activity and potent in vivo elimination of HIV-infected cells in a humanized mouse model. Sci Transl Med 11(504).

5. Herzig E, et al. (2019) Attacking Latent HIV with convertibleCAR-T Cells, a Highly Adaptable Killing Platform. Cell 179(4):880-894 e810.

6. Martinez DR, et al. (2020) Maternal Broadly Neutralizing Antibodies Can Select for Neutralization-Resistant, Infant-Transmitted/Founder HIV Variants. mBio 11(2).

7. Wu Y, et al. (2019) Rapid Elimination of Broadly Neutralizing Antibodies Correlates with Treatment Failure in the Acute Phase of Simian-Human Immunodeficiency Virus Infection. $J$ Virol 93(20).

8. Qi J, Ding C, Jiang X, \& Gao Y (2020) Advances in Developing CAR T-Cell Therapy for HIV Cure. Front Immunol 11:361.

9. Lisziewicz J, et al. (1999) Control of HIV despite the discontinuation of antiretroviral therapy. N Engl J Med 340(21):1683-1684.

10. Gupta RK, et al. (2020) Evidence for HIV-1 cure after CCR5Delta32/Delta32 allogeneic haemopoietic stem-cell transplantation 30 months post analytical treatment interruption: a case report. Lancet HIV 7(5):e340-e347.

11. Dash Pea (2019) Sequential LASER ART and CRISPR treatments lead to undetectable HIV-1 in a subset of infected humanized mice. Nature Communications Under Review January 2019.

12. Roberts JD, Bebenek K, \& Kunkel TA (1988) The accuracy of reverse transcriptase from HIV-1. Science 242(4882):1171-1173.

13. Fraser C, Hollingsworth TD, Chapman R, de Wolf F, \& Hanage WP (2007) Variation in HIV-1 set-point viral load: epidemiological analysis and an evolutionary hypothesis. Proc Natl Acad Sci U S A 104(44):17441-17446.

14. Kuiken C, Korber B, \& Shafer RW (2003) HIV sequence databases. AIDS Rev 5(1):5261.

15. Taylor BS, Sobieszczyk ME, McCutchan FE, \& Hammer SM (2008) The challenge of HIV-1 subtype diversity. N Engl J Med 358(15):1590-1602.

16. Gartner MJ, Roche M, Churchill MJ, Gorry PR, \& Flynn JK (2020) Understanding the mechanisms driving the spread of subtype C HIV-1. EBioMedicine 53:102682.

17. Liao HK, et al. (2015) Use of the CRISPR/Cas9 system as an intracellular defense against HIV-1 infection in human cells. Nat Commun 6:6413.

18. Wang G, Zhao N, Berkhout B, \& Das AT (2016) CRISPR-Cas9 Can Inhibit HIV-1 Replication but NHEJ Repair Facilitates Virus Escape. Mol Ther 24(3):522-526.

19. Wang Q, et al. (2018) Genome scale screening identification of SaCas9/gRNAs for targeting HIV-1 provirus and suppression of HIV-1 infection. Virus Res 250:21-30.

20. Gao Z, Fan M, Das AT, Herrera-Carrillo E, \& Berkhout B (2020) Extinction of all infectious HIV in cell culture by the CRISPR-Cas12a system with only a single crRNA. Nucleic Acids Res 48(10):5527-5539.

21. Ophinni Y, Inoue M, Kotaki T, \& Kameoka M (2018) CRISPR/Cas9 system targeting regulatory genes of HIV-1 inhibits viral replication in infected T-cell cultures. Sci Rep 8(1):7784.

22. Zhu W, et al. (2015) The CRISPR/Cas9 system inactivates latent HIV-1 proviral DNA. Retrovirology 12:22. 
23. Ophinni Y, Miki S, Hayashi Y, \& Kameoka M (2020) Multiplexed tat-Targeting CRISPRCas9 Protects T Cells from Acute HIV-1 Infection with Inhibition of Viral Escape. Viruses 12(11).

24. Dampier W, et al. (2017) Designing broad-spectrum anti-HIV-1 gRNAs to target patientderived variants. Sci $\operatorname{Rep} 7(1): 14413$.

25. Sullivan NT, et al. (2019) Novel gRNA design pipeline to develop broad-spectrum CRISPR/Cas9 gRNAs for safe targeting of the HIV-1 quasispecies in patients. Sci Rep 9(1):17088.

26. Stephenson KE, et al. (2020) Comparison of shortened mosaic HIV-1 vaccine schedules: a randomised, double-blind, placebo-controlled phase 1 trial (IPCAVD010/HPX1002) and a preclinical study in rhesus monkeys (NHP 17-22). Lancet HIV 7(6):e410-e421.

27. Malim MH \& Emerman M (2001) HIV-1 sequence variation: drift, shift, and attenuation. Cell 104(4):469-472.

28. de Azevedo SSD, et al. (2017) Highly divergent patterns of genetic diversity and evolution in proviral quasispecies from HIV controllers. Retrovirology 14(1):29.

29. Dash PK, et al. (2019) Sequential LASER ART and CRISPR Treatments Eliminate HIV-1 in a Subset of Infected Humanized Mice. Nat Commun 10(1):2753.

30. Ueda S, Ebina H, Kanemura Y, Misawa N, \& Koyanagi Y (2016) Anti-HIV-1 potency of the CRISPR/Cas9 system insufficient to fully inhibit viral replication. Microbiol Immunol 60(7):483-496.

31. Lebbink RJ, et al. (2017) A combinational CRISPR/Cas9 gene-editing approach can halt HIV replication and prevent viral escape. Sci Rep 7:41968.

32. Darcis G, et al. (2019) The Impact of HIV-1 Genetic Diversity on CRISPR-Cas9 Antiviral Activity and Viral Escape. Viruses 11(3).

33. Pausch P, et al. (2020) CRISPR-CasPhi from huge phages is a hypercompact genome editor. Science 369(6501):333-337.

34. Xu L, et al. (2019) CRISPR-Edited Stem Cells in a Patient with HIV and Acute Lymphocytic Leukemia. N Engl J Med 381(13):1240-1247.

35. Xiao Q, et al. (2019) CCR5 editing by Staphylococcus aureus Cas9 in human primary CD4(+) T cells and hematopoietic stem/progenitor cells promotes HIV-1 resistance and CD4(+) T cell enrichment in humanized mice. Retrovirology 16(1):15.

36. Chung $\mathrm{CH}$, et al. (2020) Safe CRISPR-Cas9 Inhibition of HIV-1 with High Specificity and Broad-Spectrum Activity by Targeting LTR NF-kappaB Binding Sites. Mol Ther Nucleic Acids 21:965-982.

37. Kaminski R, et al. (2016) Elimination of HIV-1 Genomes from Human T-lymphoid Cells by CRISPR/Cas9 Gene Editing. Sci Rep 6:22555.

38. Aubert M, et al. (2020) Gene editing and elimination of latent herpes simplex virus in vivo. Nat Commun 11(1):4148.

39. Jiang C, et al. (2020) Distinct viral reservoirs in individuals with spontaneous control of HIV-1. Nature 585(7824):261-267.

40. Sessions KJ, et al. (2020) Analysis of CRISPR/Cas9 Guide RNA Efficiency and Specificity Against Genetically Diverse HIV-1 Isolates. AIDS Res Hum Retroviruses 36(10):862-874.

41. Tsai SQ, et al. (2017) CIRCLE-seq: a highly sensitive in vitro screen for genome-wide CRISPR-Cas9 nuclease off-targets. Nat Methods 14(6):607-614.

42. Tsai SQ, et al. (2015) GUIDE-seq enables genome-wide profiling of off-target cleavage by CRISPR-Cas nucleases. Nat Biotechnol 33(2):187-197. 